\title{
Cryptanalysis of a Pseudorandom Generator for Cross-Border E-Commerce
}

\author{
Lelai Shi ${ }^{1}$, Suhui $\mathrm{Liu}^{2 *}$, Slobodan Petrović ${ }^{3}$ \\ ${ }^{1}$ Center for Industrial Economic Studies, School of Economics, Wuhan Textile University, Wuhan 430200, China \\ ${ }^{2}$ School of Mathematics and Physics, Wuhan Institute of Technology, Wuhan 430205, China \\ ${ }^{3}$ Norwegian Information Security Laboratory, Norwegian University of Science and Technology, Teknologiveien 22,2815 \\ Gjøvik, Norway
}

Corresponding Author Email: $17120801 @$ wit.edu.cn

https://doi.org/10.18280/isi.240401

Received: 28 March 2019

Accepted: 21 July 2019

\section{Keywords:}

cryptanalysis, linear feedback shift registers (LFSRS), cascade, irregular clocking, constrained edit distance

\begin{abstract}
In this paper, we study ciphertext-only cryptanalysis of a cascade of pseudorandom sequence generators employing linear feedback shift registers (LFSRs) with so-called irregular clocking. The cascade of LFSRs is a well-known pseudorandom generator scheme that produces sequences with good cryptographic characteristics (long period, high linear complexity, good statistical properties, etc.) A method of cryptanalysis of cascades containing two such LFSRs is well known. We generalize this method to cryptanalysis of a cascade with an arbitrary number of LFSRs. We reconstruct a set of candidate clock control sequences at each stage of the cascade, instead of enumerating all the possible initial states of the corresponding subcascade. The reconstruction is performed by means of an independent search through the edit distance matrix associated with every stage of the cascade. The experimental results show that such a generalized method of cryptanalysis is feasible. This topic is of great significance to the study of the security of such schemes applied to digital communications of cross-border e-commerce.
\end{abstract}

\section{INTRODUCTION}

Stream ciphers represent the backbone of security of today's most important digital communication of cross-border ecommerce. With irregularly clocked LFSRs in general and especially with their cascades, it is possible to achieve extremely long periods and large linear complexities of keystream sequences. Because of that, the most of today's high-grade stream ciphers are based on irregular clocking, in one form or another. By studying possibilities of cryptanalysis of such pseudorandom generator schemes, it is possible to determine the potential of securing modern digital communications with this type of devices and to reduce the exploitation of their vulnerabilities to a minimum.

In this paper, we study ciphertext-only cryptanalysis of a cascade of pseudorandom sequence generators employing linear feedback shift registers (LFSRs) with so-called irregular clocking. The cascade structure represents a generalization of a primitive irregular clocking structure with 2 LFSRs, of which one generates clock pulses for the other (see Figure 1). There are several ways of irregular clocking, which determine the type of the irregular clocking-based pseudorandom sequence generator. Examples of irregular clocking schemes include the stop-and-go generator [1], the Binary Rate Multiplier [2], the shrinking generator [3] and the alternating step generator [4]. It is well-known that the two most important cryptographic quality criteria for stream ciphers are the length of the period of the output sequence and the linear complexity of the output sequence [5]. Long periods and high linear complexities with structures employing LFSRs can be achieved in several ways. We can use non-linear filters [6], non-linear combiners [7] or irregular clocking. Non-linear filters and non-linear combiners were shown to be vulnerable to various kinds of attacks such as algebraic attacks $[8,9]$ and correlation attacks [10]. Besides, with irregular clocking it is possible to achieve longer periods and higher linear complexities than with non-linear filters and combiners [11]. Because of that, it is of particular interest to investigate the difficulties of cryptanalysis of such schemes.

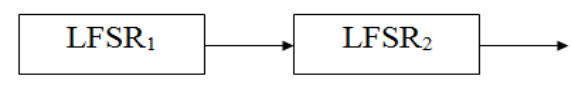

Figure 1. Elementary cascade of two LFSRs

In cryptanalysis of irregularly clocked LFSRs in general and their cascades in particular, it is not possible to directly implement correlation attack methods as used by Siegenthaler [10]. The reason for this is the need to compare the output sequence of the generator with internal sequences that are in general longer than the output sequence. Because of that, the Hamming distance measure, used by Siegenthaler has to be replaced by a more convenient measure, with which we can compare sequences of different lengths. One possibility is to use the constrained edit distance (or constrained Levenshtein distance) $[12,13]$. Thus, it is possible to use the ideas from [10] in the cryptanalysis of the pseudorandom generator schemes that employ irregularly clocked LFSRs.

In this paper, we consider stream ciphers, a large class of so-called symmetric ciphers, and we concentrate on cryptanalysis of cascades of irregularly clocked LFSRs (Figure 3) realized through the Binary Rate Multiplier (BRM) [2]. We now formalize the task of cryptanalysis of such a scheme: 
Given the prefix of the intercepted output sequence of length $M$, determine the initial states of all the LFSRs in the cascade. The goal of the paper is to determine how this task could be solved.

The decimation of sequences happens when the output sequence of a subgenerator is fed into the clock control input of one or more other subgenerators. The minimal example of such a scheme is a scheme in which one LFSR clocks another. In this paper, we generalize the ideas from Ref. [14] to cryptanalysis of cascades of irregularly clocked LFSRs.

\section{A PARTICULAR STATISTICAL MODEL APPLIED IN CRYPTANALYSIS OF CASCADE OF LFSRS}

The statistical model that we are interested in is based on the binary rate multiplier. This model was originally mentioned in Ref. [15]. The model is applied in Ref. [14] for the cryptanalysis of pseudorandom sequence generators. It is shown in Figure 2. In this paper, we analyze a stage of the cascade using this model.

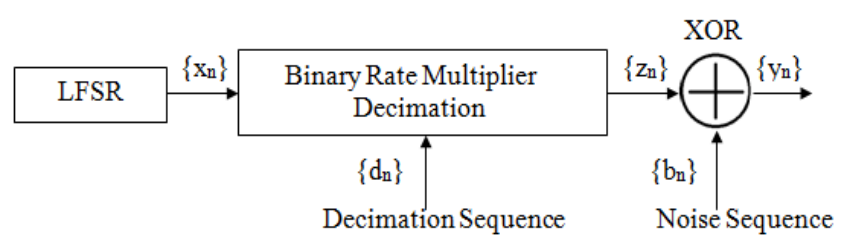

Figure 2. The statistical model of a stage of the cascade

According to Golić and Mihaljević's reaearch [15], the feedback polynomial of the LFSR in this model is given as:

$$
f(x)=1+a_{1} x+a_{2} x^{2}+\cdots+a_{n} x^{L}
$$

where, $L$ is the given length of this LFSR. $\left\{x_{n}\right\}$ is the output sequence of the LFSR. $\left\{d_{n}\right\}$, serving as the decimation sequence, is the output sequence from another LFSR. $\left\{z_{n}\right\}$ is the output sequence after the decimation processor. $\left\{b_{n}\right\}$ is the binary noise sequence, for example, the plaintext. $\left\{y_{n}\right\}$ is produced by the sum modulo 2 of the decimated sequence $\left\{z_{n}\right\}$ and the noise sequence $\left\{b_{n}\right\}$.

$$
\begin{gathered}
y_{n}=Z_{n} \bigoplus b_{n} \\
z_{n}=x_{f(n)} \\
f(n)=n+\sum_{i=0}^{n} d_{i}, n=0,1,2, \ldots
\end{gathered}
$$

In this statistical model, it is supposed that $\left\{d_{n}\right\}$ is the realization of the sequence $\left\{D_{n}\right\}$ of independent and identically distributed random variables, with a certain probability $\operatorname{Pr}\left(D_{n}=i\right)$, which should be chosen in our research as it is described in Section 3.

The binary noise sequence $\left\{b_{n}\right\}$ is the realization of the sequence of random independent and identically distributed variables $\left\{B_{n}\right\}$ with probability:

$$
\operatorname{Pr}\left(B_{n}=1\right)=\mathrm{p}<0.5, \forall \mathrm{n}
$$

\section{CRYPTANALYSIS OF THE CASCADE WITH AN ARBITRARY NUMBER OF LFSRS}

\subsection{The phases of cryptanalysis of cascade with two LFSRs}

Two phases of cryptanalysis of cascade with two LFSRs (Figure 1) are discussed in Ref. [14]. In this cascade, there are two LFSRs. The first register $\mathrm{LFSR}_{1}$ is regularly clocked while the second one $\mathrm{LFSR}_{2}$ is irregularly clocked with the clock signals from the output sequence of $\mathrm{LFSR}_{1}$ (Figure 1). The clocking satisfies the model described in Figure 2.

As it can be seen in Figure 2, the output sequence of LFSR1 $\left\{d_{n}\right\}$ serves as the decimation sequence. The output sequence of $\operatorname{LFSR}_{2}\left\{x_{n}\right\}$ is decimated by $\left\{d_{n}\right\}$. The output sequence of the binary rate multiplier $\left\{z_{n}\right\}$ and the noise sequence $\left\{b_{n}\right\}$ are summed modulo 2 to produce the final output sequence $\left\{y_{n}\right\}$.

There are two main phases in the cryptanalysis of the cascade with two LFSRs. The two phases are briefly described in Ref. [14], in which the emphasis is put on the reconstruction of suboptimal paths in the constrained edit distance array.

The first phase of the cryptanalysis of a cascade with two LFSRs is that the candidate initial states of $\mathrm{LFSR}_{2}$ should be determined. Golić and Mihaljević [15] describes a correlation attack based on the statistical model given in Section 2. Firstly, certain number of initial states are chosen at random from all the non-zero initial states of $\mathrm{LFSR}_{2}$. Then, these selected initial states are used to generate $\left\{x_{n}\right\}$, whose length depends on the length of the intercepted sequence. After that, the constrained edit distance between the intercepted sequence and each generated $\left\{x_{n}\right\}$ is calculated. The threshold is chosen to be an integer greater than or equal to the maximum of the results of computation of the constrained edit distance. In the next step, we use all the remaining initial states of $\mathrm{LFSR}_{2}$ to generate $\left\{x_{n}\right\}$, between which and the intercepted sequence, a new set of constrained edit distances is calculated. The states producing $\left\{x_{n}\right\}$, whose constrained edit distance to the intercepted output sequence is less than the threshold represent the candidate initial states for $\mathrm{LFSR}_{2}$.

The second phase of the attack is to reconstruct the clock control sequence of $\mathrm{LFSR}_{2}$. In 2007, Petrovic and FusterSabater [14] proposed an attack, in which a new algorithm was used to search for the optimal and suboptimal paths through the matrix of constrained edit distances, each corresponding to a possible clock control sequence for $\mathrm{LFSR}_{2}$. This search is directed by increasing the tolerance for weight discrepancy between the weight (i.e. constrained edit distance) of a reconstructed suboptimal path and the weight of the optimal path. In this paper, we extend the ideas from Ref. [14] to cryptanalysis of a cascade of irregularly clocked LFSRs containing an arbitrary number of LFSRs.

\subsection{The method of splitting the cascade into subcascades}

The scheme of a cascade of LFSRs presented can be divided into two parts: the first one is the subcascade that generates the clock control sequence for the last LFSR, and the second one is the last LFSR in the cascade itself (as shown in Figure 3 below).

If the prefix of the output sequence of the last LFSR is known to the attacker, it is possible to reconstruct the initial state of the last LFSR with a generalized correlation attack. It is possible to obtain a set of candidate initial states of that LFSR, which could generate the intercepted output sequence. This can be realized by using the model described in Section 
2.

The next step is to determine which initial state, among all the candidate initial states obtained, can generate the intercepted output sequence. So the control sequence that, together with one of the candidate initial states of the last LFSRs in the cascade, could generate the prefix of the intercepted sequence has to be determined. We use the constrained edit distance matrix associated with each candidate initial state to reconstruct the paths, each of which maps to a possible control sequence.

After the possible clock control sequences of the last LFSR in the cascade are reconstructed, we can treat the rest of the scheme as a cascade of lower dimension. We can use the possible clock control sequences of the last LFSR as the output sequence of the subcascade. Then the same process can be used in the subcascade.

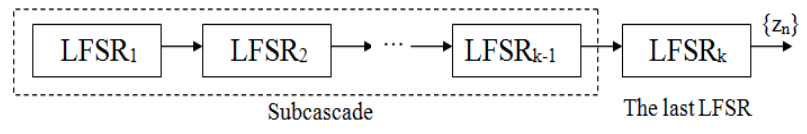

Figure 3. The scheme of a cascade of irregularly clocked LFSRs divided into two parts

\section{EXPERIMENTAL WORK}

\subsection{Objective of the experiment}

The purpose of our experiment was to verify that the methods discussed in the previous sections can be used in cryptanalysis of a cascade of LFSRs. A similar experiment with two LSFRs was implemented in Ref. [14]. In our experiment, by using the theory discussed in the previous sections, we carry out cryptanalysis of a cascade with three LSFRs. By doing this, the method is extended from the cascade with two LFSRs to that with three LFSRs. Then the same procedure can be applied to extending to the cascade with an arbitrary number of LSFRs in the future work.

We chose three LFSRs constituting the cascade (see Figure 4). These LFSRs are clocked in the following way:

$\mathrm{LFSR}_{1}$ is regularly clocked.

If the output of $\mathrm{LFSR}_{1}$ is $0, \mathrm{LFSR}_{2}$ is clocked once, and the output bit of $\mathrm{LFSR}_{2}$ is valid and is used to clock $\mathrm{LFSR}_{3}$.

If the output of $\mathrm{LFSR}_{1}$ is $1, \mathrm{LFSR}_{2}$ is clocked twice. The first output bit of $\mathrm{LFSR}_{2}$ is discarded. The second output bit is valid and is sent to clock $\mathrm{LFSR}_{3}$.

The same procedure is applied in clocking $\mathrm{LFSR}_{3}$ by using the valid output bit of $\mathrm{LFSR}_{2}$.

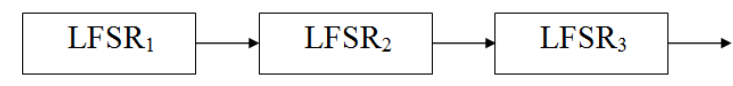

Figure 4. A cascade of three LFSRs

In our experiment, as a matter of convenience, we chose three LFSRs with the same feedback polynomials:

$$
f(x)=1+x^{2}+x^{7}+x^{6}+x^{10}
$$

Each LFSR had 4 feedback taps and 1 output tap. The output tap was the content of the leftmost cell of each LFSR. In addition, all the LFSRs had the length of 10 .

\subsection{Simulation of generating the intercepted sequence}

The aim of the attack was, given the intercepted sequence (the output sequence of $\mathrm{LFSR}_{3}$ with a certain level of noise), to determine the initial states of $\mathrm{LFSR}_{3}, \mathrm{LFSR}_{2}$ and $\mathrm{LFSR}_{1}$ successively as well as the correct clocking sequences of $\mathrm{LFSR}_{3}$ and $\mathrm{LFSR}_{2}$. In order to demonstrate our attack procedure to be feasible, we needed to compare the initial states and the clocking sequences we obtained from our attack to the correct ones, which were known in advance. This could be realized by the following logical steps:

1. The initial states of each LFSR in the cascade were chosen at random. They were recorded.

2. With the initial states obtained in the step 1 and the clocking algorithm, we could start the simulation procedure. By simulating the irregular clocking procedure, the intercepted sequence was generated.

3. The corresponding output sequences of $\mathrm{LFSR}_{2}$ and $\mathrm{LFSR}_{1}$, which were used to generate the intercepted sequence, were recorded.

4. The attack (cryptanalysis) procedure was started bearing in mind the fact that the only known information was the intercepted sequence and the feedback polynomials of each LFSR.

5. Compare the results of the attack, including the initial states of the three LFSRs and the output sequences of $\mathrm{LFSR}_{3}$ and $\mathrm{LFSR}_{2}$, to the recorded ones in step 1 and 3 .

6. If the values of each comparison are identical, the attack method is proved to be feasible.

In the simulation procedure of our experiment, the initial states of $\mathrm{LFSR}_{1}, \mathrm{LFSR}_{2}$ and $\mathrm{LFSR}_{3}$ were set to 0101111111 , 0011100100 and 1011100000 , respectively. The length of the output intercepted sequence was set to 200. The LFSRs had feedback polynomials (1). Then the simulation was executed and we generated 200 bits of the intercepted sequence $Y$ as well as the corresponding output sequence of $\mathrm{LFSR}_{1}$ (clock 1) and $\mathrm{LFSR}_{2}$ (clock 2), and the intercepted sequence $\left(\mathrm{Y}_{1}\right)$ without noise.

\subsection{Cryptanalysis processes and results}

The cryptanalysis (attack) procedure consists of the following steps:

1. Determine the candidate initial states of $\mathrm{LFSR}_{3}$.

2. Determine the correct clocking sequence of $\mathrm{LFSR}_{3}$.

3. Determine the candidate initial states of $\mathrm{LFSR}_{2}$.

4. Determine the correct clocking sequence of $\mathrm{LFSR}_{2}$.

5. Determine the correct initial state of LFSR 1 .

In this cryptanalysis procedure, it is supposed that the feedback polynomials are known in advance as shown in polynomials (1). In the first place, the method used in the realization of step 1 and step 3 was described in Ref. [15]. Furthermore, after we obtained the clocking sequence of $\mathrm{LFSR}_{2}$ in the step 4, we can calculate the initial state of $\mathrm{LFSR}_{1}$ by solving the system of linear equations in the step 5 . So, in our experiment, we shall only focus on the step 2 and the step 4.

In the step 1, with the knowledge of the intercepted sequence, we can determine a set of candidate initial states of $\mathrm{LFSR}_{3}$ (see the correlation attack described in Ref. [15]). In our experiment, we supposed that we obtained the correct initial state of $\mathrm{LFSR}_{3}$ which, together with the corresponding clocking sequence of $\mathrm{LFSR}_{3}$, could produce the intercepted sequence. 
In the step 2, the initial state of $\mathrm{LFSR}_{3}$ was set to 1011100000, which was equal to the value used in the simulation process. And the intercepted sequence used to establish the constrained edit distance was also set to be equal to the value obtained by the simulation process. The length of the intercepted sequence was set to 200 . Besides, the length $\mathrm{pl}$ out of the clocking sequence of $\mathrm{LFSR}_{3}$ was set to 40, which means only the first 40 bits of clock 2 were used in the comparison process. Then we generated the corresponding constrained edit distance matrix and reconstructed all the optimal and sub-optimal paths using algorithm in Ref. [16]. The result shows that, after searching 7612 paths, the correct clock sequence is found equal to clock 2 .

The same process was applied in the step 4, where the initial state of $\mathrm{LFSR}_{2}$ was set to 0011100100 . As the feedback polynomial of $\mathrm{LFSR}_{1}$ was known, the length pl was set to 10 , which was equal to the length of $\mathrm{LFSR}_{1}$. The length of clock 2 needed was set to 40 . Then we generated the corresponding constrained edit distance matrix and reconstructed the optimal and sub-optimal paths using algorithm in Ref. [16] again. The result shows that, after searching only 20 paths, the correct clock-control sequence was found equal to clock 1 .

The experiment results proved that the theory discussed in previous sections and the methods described in this section were feasible.

\section{CONCLUSION}

In this paper, the cryptanalysis of the cascade of irregularly clocked linear feedback shift registers is described. The attack is essentially a method for reconstruction of the initial state of the subcascade that generates the clock control sequence. The statistical model based on binary rate multiplier, which employs the constrained edit distance is used. Instead of checking all the possible initial states of the subcascade, all the possible optimal paths in the edit distance matrix as well as the suboptimal paths, whose weight-difference from the optimal ones dose not overcome the discrepancy D given in advance, are reconstructed by depth-first search. Experimental results show that the methods described in this paper, which is used to cryptanalyze a cascade with an arbitrary number of LFSRs, are applicable.

The experiment results proved that it is feasible to generalize the correlation attack against a scheme with 2 LFSRs, of which one irregularly clocks another, to a cascade of irregularly clocked LFSRs. First of all, the cascade with an arbitrary number of LFSRs was split into subcascades. Then, the possible candidate initial states of the last LFSR were reconstructed with a generalized correlation attack. Once the set of candidate initial states is known, the element that generates the intercepted output sequence has to be determined. The attack continued by determining the clock control sequence that, together with one of the candidate initial states of the last LFSR, could generate the intercepted sequence. This can be achieved by searching through the constrained edit distance matrix associated with every candidate initial state obtained in the previous step of attack. The optimal and suboptimal paths were reconstructed in the searching procedure. Once the possible clock control sequences of the last LFSR in the cascade were reconstructed, we treated the rest of the scheme as a cascade of lower dimension utilizing the reconstructed clock sequences as its output sequence. Then, the same analytical methods were applied into the cascade of lower dimension. This process continues until the possible initial states of the first LFSR in the cascade were reconstructed.

\section{REFERENCES}

[1] Beth, T., Piper, F. (1984). The stop-and-go-generator. Proceeding of EUROCRYPT 84, LNCS 209, Springer Verlag, Berlin, 88-92. https://doi.org/10.1007/3-54039757-4_9

[2] Chambers, W.G., Jennings, S.M. (1984). Linear equivalence of certain BRM shift-register sequences. Electronics Letters, 10(24): 1018-1019. https://doi.org/10.1049/el:19840693

[3] Coppersmith, D., Krawczyk, H., Mansour, Y. (1993). The shrinking generator. Advances in Cryptology CRYPTO' 93, Lecture Notes in Computer Science, Springer, Berlin, Heidelberg, 773: 22-39. https://doi.org/10.1007/3-540-48329-2_3

[4] Günther, C.G. (1987). Alternating step generators controlled by de Bruijn sequences. EUROCRYPT'87 Proceedings of the 6th annual international conference on Theory and application of cryptographic techniques. Springer Verlag, Berlin, Heidelberg, pp. 5-14. https://doi.org/10.1007/3-540-39118-5_2

[5] Menezes, A., VanOorschot, P., Vanstone, S. (1997). Handbook of applied cryptography. CRC Press. http://dx.doi.org/10.1201/9781439821916

[6] Rueppel, R.A. (1986). Analysis and design of stream ciphers. Springger-Verlag. https://doi.org/10.1007/9783-642-82865-2

[7] Beker, H., Piper, F. (1982). Cipher systems: The protection of communications. Northwood Books. https://doi.org/10.1112/blms/15.5.521

[8] Krause, M., Armknecht, F. (2003). Algebraic attacks on combiners with memory. Advances in Cryptology CRYPTO 2003, 23rd Annual International Cryptology Conference, Santa Barbara, California, USA. https://doi.org/10.1007/978-3-540-45146-4_10

[9] Courtois, N. (2003). Fast algebraic attacks on stream ciphers with linear feedback. Proceedings of Crypto 2003, LNCS 2729, Springer Verlag, pp. 176-194. https://doi.org/10.1007/978-3-540-45146-4_11

[10] Siegenthaler, T. (1985). Decrypting a class of stream ciphers using ciphertext only. IEEE Transactions on Computers, C34(1): 81-85. http://dx.doi.org/10.1109/TC.1985.1676518

[11] Gollman, D., Chambers, W.G. (1989). Clock-controlled shift registers: A review. IEEE Journal on Selected Areas in Communications, 7(4): 525-533. https://doi.org/10.1109/49.17716

[12] Oommen, B.J. (1986). Constrained string editing. Elsevier Science Inc, 40(3): 267-284. https://doi.org/10.1016/0020-0255(86)90061-7

[13] Oommen, B.J. (1987). Recognition of noisy subsequences using constrained edit distance. IEEE Transactions on Pattern Analysis \& Machine Intelligence, 9(5):

676-685. https://doi.org/10.1109/TPAMI.1987.4767962

[14] Petrović, S., Fúster, A. (2007). Reconstruction of suboptimal paths in the constrained edit distance array with application in cryptanalysis with application in cryptanalysis. Proceedings of the 2007 international 
conference on computational science and its applications, ICCSA 2007, Kuala Lumpur, Malaysia, Lecture Notes in Computer Science, LNCS 4707, Part III: 597-610. http://dx.doi.org/10.1007/978-3-540-74484-9_52

[15] Golić, J., Mihaljević, M. (1991). A generalized correlation attack on a class of stream ciphers based on the Levenshtein distance. Journal of Cryptology, 3(3): 201-212. https://doi.org/10.1007/BF00196912
[16] Petrović, S., Fúster, A. (2004). Clock control sequence reconstruction in the ciphertext only attack scenario. International Conference on Information and Communications Security, Lecture Notes in Computer Science, Springer, Berlin, Heidelberg, 3269: 427-439. https://doi.org/10.1007/978-3-540-30191-2_33 